\title{
Association between obesity and heart rate variability indices: an intuition toward cardiac autonomic alteration - a risk of CVD
}

\author{
This article was published in the following Dove Press journal: \\ Diabetes, Metabolic Syndrome and Obesity:Targets and Therapy \\ 17 February 2017 \\ Number of times this article has been viewed
}

\section{Ram Lochan Yadav' \\ Prakash Kumar Yadav' \\ Laxmi Kumari Yadav² \\ Kopila Agrawal ${ }^{3}$ \\ Santosh Kumar Sah ${ }^{4}$ \\ Md Nazrul Islam' \\ 'Department of Physiology, \\ 2Department of Microbiology, \\ Chitwan Medical College, Bharatpur, ${ }^{3}$ Department of Physiology, BP Koirala Institute of Health Sciences, Dharan, ${ }^{4}$ Department of Biochemistry, Janaki Medical College, Janakpur, Nepal}

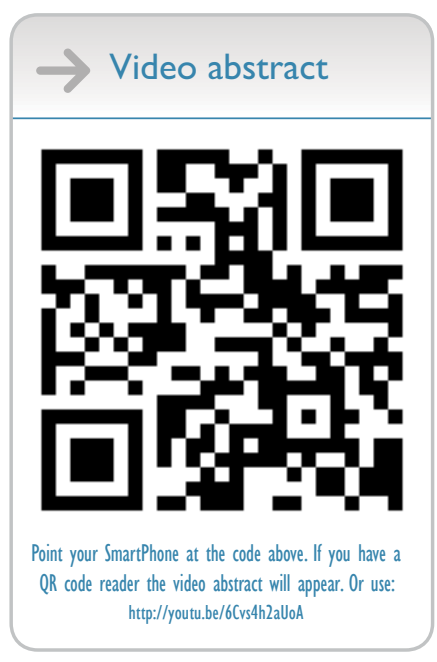

Correspondence: Ram Lochan Yadav Department of Physiology, Chitwan Medical College, Bharatpur-13, 44200 , Nepal

Tel +9779848096590

Email dr.ramlochan04@gmail.com
Background: Obese people have a higher prevalence of cardiovascular disease, which is supposed to be due to autonomic dysfunction and/or metabolic disorder. The alterations in cardiac autonomic functions bring out the changes in the heart rate variability (HRV) indicators, an assessing tool for cardiac autonomic conditions.

Objective: To compare the cardiac autonomic activity between obese and normal weight adults and find out the highest association between the indices of HRV and obesity.

Methods: The study was conducted in 30 adult obese persons (body mass index [BMI] $>30 \mathrm{~kg} / \mathrm{m}^{2}$ ) and 29 healthy normal weight controls (BMI $18-24 \mathrm{~kg} / \mathrm{m}^{2}$ ). Short-term HRV variables were assessed using standard protocol. Data were compared between groups using Mann-Whitney $U$ test. Obesity indices such as waist circumference, hip circumference, waisthip ratio (WHR), and BMI were measured and calculated, and they were correlated with HRV indices using Spearman's correlation analysis.

Results: In the obese group, there was a significant increase in the mean heart rate, whereas the HRV parasympathetic indicators were less (eg, root mean square of differences of successive RR intervals $[28.75\{16.72-38.35\}$ vs $41.55\{30.6-56.75\} \mathrm{ms}, p=0.018]$, number of RR intervals that differ by $>50 \mathrm{~ms}$, that is, $\mathrm{NN} 50[15.5\{2-39\}$ vs $83.5\{32.75-116.25\}, p=0.010]$, etc) and the sympathetic indicator low frequency (LF)/high frequency (HF) ratio (1.2 [0.65-2.20] vs 0.79 [0.5-1.02], $p=0.045$ ) was more than that of the normal weight group. Spearman's correlation between HRV and obesity indices showed significant positive correlation of WHR with LF in normalized unit $(r=0.478, p<0.01)$ and LF/HF ratio $(r=0.479, p<0.01)$, whereas it had significant negative correlation with high frequency power $\mathrm{ms}^{2}(r=-0.374, p<0.05)$ and HF in normalized unit $(r=-0.478, p<0.01)$. There was a nonsignificant correlation of BMI with HRV variables in obese individuals.

Conclusion: Increased WHR, by far an indicator of visceral adiposity, was strongly associated with reduced cardiac parasympathetic and increased sympathetic activity in obese individuals defined by BMI. However, BMI itself has a weak relationship with HRV cardiac autonomic markers. Thus, even with a slight increase in WHR in an individual, there could be a greater risk of cardiovascular morbidity and mortality brought about by cardiac autonomic alterations. Keywords: obesity, heart rate variability, HRV, body mass index, BMI, waist-hip ratio, WHR, cardiac autonomic regulation

\section{Introduction}

Obesity is one of the leading disorders that increases the all-cause mortality in patients with increased body mass index (BMI), especially causing death from cardiovascular 
disease (CVD). ${ }^{1}$ Despite the relatively consistent findings of increased prevalence of CVD in obesity, the reason for these associations remains obscure. Many factors have been suggested as causes for this relationship, such as insulin resistance, hypertension, and reduced high-density lipoprotein. However, it has also been suggested that a reduction in autonomic function might be the mechanism for the increased prevalence of CVD in obesity. ${ }^{2}$ Recent studies have concentrated on the autonomic activity of the heart itself reported controversial findings. ${ }^{3}$ The autonomic nervous system is a control system that acts largely unconsciously and regulates bodily functions such as the heart rate, digestion, respiratory rate, pupillary response, urination, and sexual arousal through its two branches: the sympathetic and parasympathetic nervous systems. ${ }^{4}$ Heart rate variability (HRV) measures the effect of autonomic function on the heart alone. ${ }^{5}$ Even a slight variation in autonomic regulation of the heart changes the heart rate and rhythm. The HRV looks through beat-tobeat variation during electrocardiogram (ECG) recording. Therefore, it could be the most useful and the simplest noninvasive method to investigate the effect of obesity on CVD. ${ }^{6}$ It is important to emphasize the effect of obesity on $\mathrm{HRV}$, as decreased HRV significantly increases cardiovascular mortality. ${ }^{7}$ Because the autonomic nervous system controls a significant part of the internal functions of the body, fat disequilibrium in obesity is an important negative factor. The risk of diseases appears to increase as a function of the percent fat content above an upper limit of normal in the body. For the purpose of risk stratification, it is convenient to have cut-off values of body fat content based upon its observed association with disease. ${ }^{8}$ The measurement of body fat is too complex to be of practical clinical application. A series of anthropometric indices were used as obesity measures in these studies, including the BMI, waist-hip ratio (WHR), waist circumference (WC), and waist stature ratio. Among them, the BMI is most widely used to categorize underweight, overweight, and obese populations. ${ }^{9}$ However, it is not yet fully clear which anthropometric index of obesity has the strongest association with cardiac autonomic markers. Therefore, this study used anthropometric obesity indices such as BMI, WC, hip circumference (HC), and WHR and HRV indices with an aim to compare the HRV between obese and normal weight adults and to establish the highest association between obesity and HRV indices.

\section{Materials and methods}

This cross-sectional comparative study was conducted in the neurophysiology laboratory of the Department of Physiology,
BP Koirala Institute of Health Sciences (BPKIHS), Nepal. Thirty normotensive obese individuals (mean age 32.07士7.25 years) and 29 age-matched normal weight controls (mean age $30.48 \pm 8.01$ years) were recruited from the medical staffs, students, and the attendants of the patients at BPKIHS. All participants were adult male. The mean height of the obese adults was $1.60 \pm 0.99 \mathrm{~m}$ and that of the controls was $1.66 \pm 0.10$ m. Mean BMI was $32.02 \pm 2.89$ and $21.87 \pm 2.40 \mathrm{~kg} / \mathrm{m}^{2}$, respectively. As per the World Health Organization directives, obese was defined as a BMI of over 30 and normal weight as a BMI of $<25$, that is, between 18 and $24 \mathrm{~kg} / \mathrm{m}^{2} .{ }^{10}$ For inclusion in the study, the subjects were required to be between 18 and 75 years of age and they also had to meet the BMI criteria presented above. Informed written consent was taken from all the subjects and they were screened for any history of drugs/ alcohol intake, familial history of hypertension and cardiac diseases, or presence of any medical illness likely to affect the HRV parameters based on clinical history and physical examination. None of the subjects reported any symptoms suggestive of peripheral or autonomic neuropathy, such as giddiness on standing, urinary urgency, tingling sensation of limbs, or limb weakness, or gastrointestinal symptoms like burning sensation in epigastric region, diarrhea, and constipation. Hypertensive persons were excluded from the study based on the diagnostic criteria of Joint National Committee-7 on the prevention, detection, evaluation, and treatment of high blood pressure. ${ }^{11}$ The choice of the patients was very selective to attribute a potential pathogenetic value to a metabolic alteration typical of the obese patients, represented by normal blood pressure level. The study was approved by the institutional ethical committee of BPKIHS, Nepal.

Room temperature of the laboratory was maintained at the thermo neutral zone, that is, $26^{\circ} \mathrm{C} \pm 2^{\circ} \mathrm{C}$. The required setup was checked before commencing the test. Furthermore, the subjects were made comfortable and familiar with the laboratory setup and conditions, and were advised to relax completely during recording. The subjects were instructed not to perform any exercise $40 \mathrm{~h}$ before the day of the experiment and to avoid drugs and caffeine $12 \mathrm{~h}$ before the test. The blood pressure of each subject was measured in supine position. Analysis of HRV was performed based on 5 min ECG recorded at rest in the supine position. Recordings were taken during 08:00 am-11:00 am to avoid any hemodynamic effect on HRV.

\section{Anthropometric measurements}

Age was recorded in complete years, that is, rounded off to the nearest years. Standing height was recorded using a stadiometer (Prestige brand) by making the subject stand 
without shoes and shocks with the feet parallel and pointing forward on a special platform to which a vertical measuring rod marked in metric scale was fixed. The subject was made to stand as tall as possible with the head positioned in Frankfort plane position (the plane joining the lower border of the left orbit and the upper margin of the external auditory meatus in a horizontal position). The head piece of the stadiometer or the sliding part of the measuring rod was lowered so that the hair (if present) was pressed flat. Height was recorded in centimeters to the resolution of the height rule (ie, the nearest quarter of a centimeter). Weight of each subject was taken on a balanced beam scale (Dr Morepen MS02B mechanical weighing machine). The scale was placed on a hard floor surface and calibrated with standardized weights at the beginning and end of each examining day. After removing their heavy outer garments (jacket, coat, trousers, skirts, wrist watch, mobile from pocket, etc), the subjects were asked to stand in the center of the platform to distribute their body weight evenly to both feet. The weight was recorded to resolution of the scale (the nearest $0.1-0.2 \mathrm{~kg}$ ). BMI was calculated from the standard formula, that is, $\mathrm{BMI}=$ weight $(\mathrm{kg}) /$ height $\left(\mathrm{m}^{2}\right)$.

\section{WC and $\mathrm{HC}$}

$\mathrm{WC}$ and $\mathrm{HC}$ were measured twice to the nearest $0.5 \mathrm{~cm}$ with a flexible, but nonelastic measuring tape. WC was measured at the level of the natural waist (the narrowest part of the torso) or one finger width below the umbilicus. HC was measured at the maximum circumference of the buttocks posteriorly and the symphysis pubis anteriorly in a horizontal plane. ${ }^{12}$ All anthropometric measures were taken in the fasting state with the participants dressed in light clothing and without shoes. WHR was calculated using the following formula:

$$
\mathrm{WHR}=\mathrm{WC}(\mathrm{cm}) / \mathrm{HC}(\mathrm{cm})
$$

\section{ECG recording and HRV analysis}

For HRV, ECG at spontaneous respiration was recorded for $5 \mathrm{~min}$ in a supine position after $15 \mathrm{~min}$ of supine rest. Blood pressure was recorded before taking the ECG recording. ECG electrolyte gel was applied on the skin underneath the standard limb leads after cleaning the skin with methyl alcohol to decrease the skin impedance so that the electrical signal from the body is conducted easily by the electrodes. The subjects were instructed to take supine rest with normal breathing, relax and close their eyes, but not to fall asleep, in order to obtain steady-state hemodynamics before commencement of the recording and during recording so that the problem of nonstationarity of the ECG signals is avoided.
The ECG was taken from one of the standard limb leads having the most prominent $\mathrm{R}$ wave. The ECG electrodes were joined to isolated ECG amplifier/coupler of Coulbourn Instruments and its software (WinDaq Pro/Pro+ model no. DI-400 series; Akron, OH, USA) in order to capture the ECG signals for HRV. The sampling frequency of the ECG was set at $2000 \mathrm{~Hz}$. The recordings were edited and corrected manually for ectopic beats, arrhythmias, noise, and trends from the WinDaq Pro/Pro+ software before any calculation of HRV. After that, "R" wave detection of normal QRS complex was completed by using WinDaq Pro/Pro+ software and the $\mathrm{R}$ wave detected by ECG was edited manually to ensure all $\mathrm{R}$ waves. Thereafter, QRS complex occurrence times were estimated using the same software and the file was saved as a Lotus file, which was readable by MS Excel. The cumulative values of $\mathrm{R}-\mathrm{R}$ intervals were converted into individual $\mathrm{R}-\mathrm{R}$ interval series in MS Excel from the Lotus file. Thus, the intervals between successive normal-to-normal QRS complexes (RR intervals resulting from sinoatrial node driven rhythm) or instantaneous heart rate values for each cardiac cycle were determined. Rechecking and editing, if required, were done manually. Thereafter, the RR intervals were saved in ASCII format. It was readable by "Kubios HRV analysis software version 2.0", which was used to calculate the time domain and frequency domain measures of HRV. This software has been developed by the biomedical signal analysis group, Department of Applied Physics, Ohio of Kuopio, Finland.

The HRV variables analyzed were: the time domain measures included standard deviation of all RR intervals (SDNN, ms), root mean square of differences of successive RR intervals (RMSSD, ms), number of RR intervals that differ by $>50 \mathrm{~ms}$ (NN50 count), and percentage of consecutive RR intervals that differ by $>50 \mathrm{~ms}$ (pNN50\%); and the frequency domain measures included low frequency power (LF ms ${ }^{2}, 0.04-0.15 \mathrm{~Hz}$ ), high frequency power (HF $\mathrm{ms}^{2}$, $0.15-0.4 \mathrm{~Hz}$ ), low frequency in normalized unit (LF nu), high frequency in normalized unit ( $\mathrm{HF}$ nu), and LF/HF - ratio of absolute LF power to HF power. ${ }^{4}$

\section{Statistical analysis}

Statistical analysis was performed with Statistical Package for the Social Sciences software (version 20; IBM Corporation, Armonk, NY, USA). Differences in variables between the groups were tested using Mann-Whitney $U$ test for the data having nonparametric distribution and Student's $t$-test for the data with parametric distribution. The results obtained from the former are expressed as median (interquartile range) and 
from the latter as mean \pm standard deviation (SD). Spearman's correlation was used to correlate the HRV measures and the obesity indices within groups. Statistical significance was considered to be $p<0.05$.

\section{Results}

Both groups were comparable in terms of their age, height, respiratory rate, and blood sugar. However, obese persons had significantly higher body weight $(p<0.001)$, BMI $(p<0.001)$, WC $(p<0.001), \mathrm{HC}(p<0.001)$, WHR $(p<0.001)$, resting pulse rate, systolic blood pressure (SBP; $p<0.05)$, and diastolic blood pressure (DBP; $p<0.001)$ than normal weight controls (Table 1). The time domain, frequency domain, and Poincare plot variables of HRV of both the groups were calculated and are presented comparatively in Table 2. Among the time domain measures, SDNN, RMSSD, NN50 count, and pNN50\% were calculated. All these time domain variables were significantly less $(p<0.05)$ in the obese group as compared to the normal weight group.

The variables analyzed among the frequency domain measures included LF power, HF power, its percentage and normalized units, and the ratio of $\mathrm{LF}$ to $\mathrm{HF}$ ( $\mathrm{LF} / \mathrm{HF}$ ratio). Of these variables, HF power $\left(\mathrm{ms}^{2}\right)\left(\mathrm{HF} \mathrm{ms}^{2}, p<0.05\right)$ and LF/ HF ratio $(p<0.05)$ were significantly higher in obese group than in normal weight controls. The other frequency domain variables were comparable between the groups. The variables analyzed in Poincare plot were SD1, SD2, and SD1/SD2 ratio (Table 2). SD1 $(p<0.05)$ was significantly less in obese group in comparison to the normal weight group.

The Spearman's correlation between HRV and the anthropometric/cardiorespiratory/biochemical variables in the obese group $(n=30)$ is presented in Table 3 . In obese subjects, age showed a significant correlation with all the HRV measures

Table I Comparison of anthropometric variables between obese and normal weight groups

\begin{tabular}{|c|c|c|c|}
\hline Variables & $\begin{array}{l}\text { Obese } \\
(n=30) \\
\text { Mean } \pm \text { SD }\end{array}$ & $\begin{array}{l}\text { Normal } \\
\text { weight }(n=29) \\
\text { Mean } \pm \text { SD }\end{array}$ & p-Value \\
\hline Age (years) & $32.07 \pm 7.25$ & $30.48 \pm 8.012$ & 0.429 \\
\hline Height (m) & $1.60 \pm 0.099$ & $1.66 \pm 0.10$ & 0.038 \\
\hline Weight (kg) & $82.93 \pm 11.14$ & $60.69 \pm 9.43$ & $<0.001$ \\
\hline BMI $\left(\mathrm{kg} / \mathrm{m}^{2}\right)$ & $30.02 \pm 2.89$ & $21.87 \pm 2.40$ & $<0.001$ \\
\hline Waist circumference $(\mathrm{cm})$ & $98.30 \pm 14.90$ & $62.97 \pm I 1.04$ & $<0.001$ \\
\hline Hip circumference $(\mathrm{cm})$ & $99.72 \pm 13.27$ & $81.97 \pm 8.38$ & $<0.001$ \\
\hline WHR & $0.98 \pm 0.06$ & $0.76 \pm 0.07$ & $<0.001$ \\
\hline $\mathrm{SBP}(\mathrm{mmHg})$ & $121.20 \pm 9.89$ & $1 \mid 3.24 \pm 11.07$ & 0.005 \\
\hline $\mathrm{DBP}(\mathrm{mmHg})$ & $84.97 \pm 7.87$ & $74.83 \pm 10.31$ & $<0.001$ \\
\hline Fasting blood sugar $(\mathrm{mg} / \mathrm{dL})$ & $84.80 \pm 17.52$ & $85.72 \pm 15.36$ & 0.830 \\
\hline
\end{tabular}

Note: Values in bold indicate statistical significance.

Abbreviations: BMI, body mass index; DBP, diastolic blood pressure; SBP, systolic blood pressure; SD, standard deviation; WHR, waist-hip ratio.
Table 2 Comparison of HRV measures between obese $(n=30)$ and normal weight $(n=29)$ groups

\begin{tabular}{|c|c|c|c|}
\hline Variables & $\begin{array}{l}\text { Obese }(n=30) \\
\text { median }(Q \mid-Q 3)\end{array}$ & $\begin{array}{l}\text { Normal weight }(n=29) \\
\text { median (QI-Q3) }\end{array}$ & $p$-Value \\
\hline SDNN (ms) & $35.55(26.77-49.25)$ & $46.15(37.22-58.57)$ & 0.038 \\
\hline RMSSD (ms) & $28.75(16.72-38.35)$ & $41.55(30.6-56.75)$ & 0.018 \\
\hline NN50 count & $15.5(2-39)$ & $83.5(32.75-116.25)$ & 0.010 \\
\hline PNN50\% & $6.4(0.5-17.33)$ & $25.65(11.42-40.5)$ & 0.012 \\
\hline LF $m s^{2}$ & $248(\mid 110.75-465)$ & $480(187-953)$ & 0.063 \\
\hline $\mathrm{HF} m s^{2}$ & $216(103.5-530)$ & $640.5(300.75-1219.75)$ & 0.014 \\
\hline LF\% & $26.15(21.6-36.55)$ & $27.85(18.62-36.92)$ & 0.921 \\
\hline HF\% & $23(12.15-45.28)$ & $34.3(23.57-45.25)$ & 0.192 \\
\hline LF nu & $54.5(39.65)$ & 44.1 (33.75-54.82) & 0.079 \\
\hline HF nu & $45.5(31.3-60.35)$ & $55.9(45.17-66.25)$ & 0.079 \\
\hline LF/HF & $1.2(0.65-2.20)$ & $0.79(0.5-1.02)$ & 0.045 \\
\hline SDI & $21.55(11.82)$ & $29.45(21.65-40.2)$ & 0.042 \\
\hline SD2 & $45.30(32.85-60.03)$ & $60.4(45-70.77)$ & 0.07 \\
\hline SDI/SD2 & $0.46(0.33-0.53)$ & $0.49(0.39-0.61)$ & 0.246 \\
\hline
\end{tabular}

Note: Values in bold indicate statistical significance.

Abbreviations: HF\%, high frequency percent; HF, high frequency; LF\%, low frequency percent; LF, low frequency; HF nu, high frequency normalized unit; HRV, heart rate variability; LF nu, low frequency normalized unit; NN50 count, number of RR intervals that differ by $>50 \mathrm{~ms}$; PNN50\%, percentage of consecutive RR intervals that differ by $>50 \mathrm{~ms}$; Q I-Q3, interquartile range; RMSSD, root mean square of differences of successive RR intervals; SDI, Poincare plot SDI; SDI/SD2, SDI:SD2 ratio; SD2, Poincare plot SD2; SDNN, standard deviation of all RR intervals.

studied except SD1/SD2 ratio. Age was found to be negatively correlated with SDNN ( $r=-0.495, p<0.01)$, RMSSD $(r=-0.473, p<0.01)$, NN50 count $(r=-0.473, p<0.01)$, PNN50\% $(r=-0.431, p<0.05)$, LF ms ${ }^{2}(r=-0.433, p<0.05)$, $\mathrm{HF} \mathrm{ms}^{2}(r=-0.557, p<0.01)$, HF nu $(r=-0.361, p<0.05), \mathrm{SD} 1$ $(r=-0.400, p<0.05)$, and SD2 $(r=-0.489, p<0.01)$, whereas it was positively correlated with low LF nu $(r=0.361, p<0.05)$ and $\mathrm{LF} / \mathrm{HF}$ ratio $(r=0.365, p<0.05)$.

There was no significant correlation of BMI with HRV variables in obese subjects. Regarding blood pressure, SBP was found to be significantly correlated in a negative direction with SDNN $(r=-0.355, p<0.05), \mathrm{HF} \mathrm{ms}^{2}(r=-0.426$, $p<0.05)$, HF nu $(r=-0.515, p<0.01)$, and SD2 $(r=-0.372$, $p<0.05$ ), whereas it was found to be positively correlated with $\mathrm{LF} \mathrm{nu}(r=0.515, p<0.01)$ and LF/HF ratio $(r=0.516, p<0.01)$. None of the anthropometric/cardiorespiratory/biochemical variables in the normal weight group correlated significantly with the HRV variables (Table 4).

\section{Discussion}

In our study, both groups were comparable in terms of their age, height, respiratory rate, and blood sugar. However, obese persons had significantly higher body weight, BMI, and WHR than normal weight controls. The resting pulse rate was significantly higher in the obese group in comparison to the normal weight group. The increase in pulse rate is in accordance with the increase in mean heart rate of the person. 
Table 3 Spearman's rank correlation between heart rate variability and anthropometric/cardiorespiratory/biochemical variables in obese group $(\mathrm{n}=30)$

\begin{tabular}{|c|c|c|c|c|c|c|c|c|c|c|}
\hline Variables & & Age & BMI & PR & SBP & DBP & WC & $\mathrm{HC}$ & WHR & FS \\
\hline \multirow[t]{2}{*}{ SDNN (ms) } & Rho & -0.495 & -0.016 & -0.661 & -0.355 & -0.287 & -0.080 & 0.008 & -0.228 & -0.206 \\
\hline & $p$-Value & 0.005 & 0.934 & 0.000 & 0.055 & 0.124 & 0.674 & 0.968 & 0.226 & 0.274 \\
\hline \multirow[t]{2}{*}{ RMSSD (ms) } & Rho & -0.473 & 0.179 & -0.625 & -0.235 & -0.225 & 0.034 & 0.068 & -0.057 & -0.323 \\
\hline & $p$-Value & 0.008 & 0.344 & 0.000 & 0.210 & 0.231 & 0.860 & 0.721 & 0.767 & 0.082 \\
\hline \multirow[t]{2}{*}{ NN50 count } & Rho & -0.473 & 0.158 & -0.638 & -0.205 & -0.166 & -0.010 & 0.029 & -0.091 & -0.311 \\
\hline & $p$-Value & 0.008 & 0.405 & 0.000 & 0.276 & 0.380 & 0.958 & 0.879 & 0.633 & 0.095 \\
\hline \multirow[t]{2}{*}{ PNN50\% } & Rho & -0.431 & 0.056 & -0.662 & -0.285 & -0.256 & -0.050 & $0.04 I$ & -0.221 & -0.205 \\
\hline & $p$-Value & 0.017 & 0.769 & 0.000 & 0.126 & 0.172 & 0.791 & 0.829 & 0.242 & 0.277 \\
\hline \multirow[t]{2}{*}{$\mathrm{LF} m \mathrm{~s}^{2}$} & Rho & -0.433 & 0.000 & -0.728 & -0.103 & -0.055 & -138 & -0.097 & -0.122 & -0.183 \\
\hline & $p$-Value & 0.017 & 1.000 & 0.000 & 0.587 & 0.773 & 0.466 & 0.610 & 0.520 & 0.332 \\
\hline \multirow[t]{2}{*}{$\mathrm{HF} \mathrm{ms}^{2}$} & Rho & -0.557 & 0.015 & -0.535 & -0.426 & -0.377 & -0.196 & -0.069 & -0.374 & -0.262 \\
\hline & $p$-Value & 0.001 & 0.939 & 0.002 & 0.019 & 0.040 & 0.299 & 0.718 & 0.042 & 0.163 \\
\hline \multirow[t]{2}{*}{ LF nu } & Rho & 0.361 & -0.006 & -0.004 & 0.515 & 0.452 & 0.172 & -0.008 & 0.478 & 0.186 \\
\hline & $p$-Value & 0.050 & 0.976 & 0.985 & 0.004 & 0.012 & 0.364 & 0.966 & 0.008 & 0.324 \\
\hline \multirow[t]{2}{*}{$\mathrm{HF}$ nu } & Rho & -0.361 & 0.006 & 0.004 & -0.515 & -0.452 & -0.172 & 0.008 & -0.478 & -0.186 \\
\hline & $p$-Value & 0.050 & 0.976 & 0.985 & 0.004 & 0.012 & 0.364 & 0.966 & 0.008 & 0.324 \\
\hline \multirow[t]{2}{*}{$\mathrm{LF} / \mathrm{HF}$} & Rho & 0.365 & -0.005 & -0.001 & 0.516 & 0.454 & 0.135 & -0.055 & 0.479 & 0.181 \\
\hline & $p$-Value & 0.047 & 0.980 & 0.994 & 0.004 & 0.012 & 0.476 & 0.772 & 0.007 & 0.339 \\
\hline \multirow[t]{2}{*}{ SDI } & Rho & -0.400 & 0.161 & -0.606 & -0.310 & -0.295 & 0.036 & 0.131 & -0.159 & -0.220 \\
\hline & $p$-Value & 0.029 & 0.396 & 0.000 & 0.096 & 0.113 & 0.849 & 0.491 & 0.402 & 0.242 \\
\hline \multirow[t]{2}{*}{ SD2 } & Rho & -0.489 & -0.082 & -0.667 & -0.372 & -0.289 & 0.080 & -0.005 & -0.218 & -0.219 \\
\hline & $p$-Value & 0.006 & 0.666 & 0.000 & 0.043 & 0.121 & 0.675 & 0.979 & 0.247 & 0.245 \\
\hline \multirow[t]{2}{*}{ SDI/SD2 } & Rho & -0.144 & 0.324 & -0.325 & -0.115 & -0.166 & 0.133 & 0.199 & -0.058 & -0.229 \\
\hline & $p$-Value & 0.448 & 0.081 & 0.080 & 0.546 & 0.381 & 0.482 & 0.291 & 0.762 & 0.224 \\
\hline
\end{tabular}

Notes: DBP in obese individuals showed a significant negative correlation with $\mathrm{HF} \mathrm{ms}^{2}(r=-0.377, p<0.05)$ and $\mathrm{HF}$ nu $(r=-0.452$, $p<0.05)$, but it showed a positive correlation with LF nu $(r=0.452, p<0.05)$ and LF/HF ratio $(r=0.454, p<0.05)$. There was a significant positive correlation of WHR with LF nu $(r=0.478, p<0.0 \mathrm{I})$ and LF/HF ratio $(r=0.479$, $p<0.0 \mathrm{I})$, whereas it had a significant negative correlation with high frequency power (HFPR) $\mathrm{ms}^{2}(r=-0.374, p<0.05)$ and $\mathrm{HF}$ nu $(r=-0.478, p<0.0 \mathrm{I})$. Values in bold indicate statistical significance.

Abbreviations: BMI, body mass index; DBP, diastolic blood pressure; FS, fasting blood sugar; HC, hip circumference; $\mathrm{HF}$, high frequency; FH nu, high frequency normalized unit; LF, low frequency; LF/HF, low frequency:high frequency ratio; LF nu, low frequency normalized unit; NN50 count, number of RR intervals that differ by $>50$ ms; PNN50\%, percentage of consecutive RR intervals that differ by $>50 \mathrm{~ms}$; RMSSD, root mean square of differences of successive RR intervals; SBP, systolic blood pressure; SDI, Poincare plot SDI; SD2, Poincare plot SD2; SDI/SD2, SDI:SD2 ratio; SDNN, standard deviation of all RR intervals; WC, waist circumference; WHR, waist-hip ratio; $\mathrm{PR}$, pulse rate (beats/min).

Several studies support the finding of tachycardia in obese people, and it is due to altered autonomic modulation of the intrinsic heart rate. ${ }^{13-16}$

Our study also confirmed that both SBP and DBP were significantly higher in the obese group as compared to normal weight controls, which is consistent with several epidemiological studies. ${ }^{17,18}$ It is estimated that as much as one-third of the hypertension may be attributable to obesity in populations where hypertension and obesity are widely prevalent. ${ }^{18}$

The HRV variables SDNN, RMSSD, NN50 count, pNN50, HF indices in milliseconds squared, and SD1, which reflect the cardiac parasympathetic nerve activity, ${ }^{4,19}$ were lower in obese persons than in normal weight persons. Moreover, as expected, the sympathetic marker $\mathrm{LF} / \mathrm{HF}$ ratio ${ }^{4,19}$ was increased in obese subjects in comparison to normal weight controls. It has been reported that obese persons have different HRV in response to some external stimuli. ${ }^{20}$ We recorded the HRV variables in subjects in resting state in order to avoid the fluctuation caused in RR intervals of ECG recording by any external stimulus to ensure that all changes that were observed in HRV in obese people were merely due to obesity.

Reports by Thorp and Schlaich, ${ }^{21}$ Laederach-Hofmann et $\mathrm{al}^{22}$ and Esler et $\mathrm{al}^{23}$ are in favor of our findings and they mentioned that obese persons suffer from an increased mortality risk supposedly due to cardiovascular disorders related to either continuously lowered parasympathetic or heightened sympathetic activation. Studies have also documented reduced HRV among overweight and obese individuals. In a study of 10 women with early-onset familial obesity and 10 nonobese women, several indices of HRV were reduced in the obese women. ${ }^{24}$ Karason et $\mathrm{al}^{25}$ studied 28 obese patients referred for gastroplasty, 24 obese patients using a lifestyle dietary modification approach, and 28 nonobese persons. At baseline, both obese groups had reduced HF values relative to the nonobese participants. After 1 year of follow-up, those persons who had undergone gastroplasty had an average weight loss of $28 \%$ and showed evidence of increased vagal 
Table 4 Spearman's correlation between HRV and anthropometric/cardiorespiratory/biochemical variables in normal weight group $(n=29)$

\begin{tabular}{|c|c|c|c|c|c|c|c|c|c|c|}
\hline Variables & & Age & BMI & PR & SBP & DBP & WC & $\mathrm{HC}$ & WHR & FS \\
\hline \multirow[t]{2}{*}{ SDNN (ms) } & Rho & 0.131 & 0.078 & 0.009 & -0.044 & -0.099 & -0.208 & -0.228 & -0.205 & 0.074 \\
\hline & $p$-Value & 0.497 & 0.688 & 0.965 & 0.823 & 0.610 & 0.279 & 0.234 & 0.287 & 0.703 \\
\hline \multirow[t]{2}{*}{ RMSSD (ms) } & Rho & -0.050 & 0.034 & 0.089 & -0.088 & -0.035 & -0.056 & -0.138 & -0.044 & -0.017 \\
\hline & $p$-Value & 0.798 & 0.862 & 0.646 & 0.649 & 0.857 & 0.773 & 0.475 & 0.821 & 0.931 \\
\hline \multirow[t]{2}{*}{ NN50 count } & Rho & 0.033 & 0.150 & 0.177 & -0.034 & 0.018 & 0.039 & -0.067 & 0.039 & -0.190 \\
\hline & $p$-Value & 0.864 & 0.437 & 0.358 & 0.860 & 0.924 & 0.843 & 0.728 & 0.839 & 0.323 \\
\hline \multirow[t]{2}{*}{ PNN50\% } & Rho & -0.024 & 0.044 & 0.078 & -0.074 & -0.018 & -0.066 & -0.151 & -0.063 & -0.076 \\
\hline & $p$-Value & 0.902 & 0.823 & 0.689 & 0.704 & 0.926 & 0.732 & 0.435 & 0.747 & 0.695 \\
\hline \multirow[t]{2}{*}{ LF $\mathrm{ms}^{2}$} & Rho & 0.040 & 0.007 & 0.029 & 0.003 & -0.015 & -0.077 & -0.081 & -0.136 & 0.102 \\
\hline & $p$-Value & 0.838 & 0.971 & 0.883 & 0.986 & 0.937 & 0.692 & 0.675 & 0.481 & 0.597 \\
\hline \multirow[t]{2}{*}{$\mathrm{HF} \mathrm{ms}^{2}$} & Rho & 0.197 & 0.055 & 0.129 & -0.021 & -0.052 & -0.259 & -0.166 & -0.302 & 0.112 \\
\hline & $p$-Value & 0.306 & 0.779 & 0.504 & 0.914 & 0.790 & 0.175 & 0.390 & 0.111 & 0.563 \\
\hline \multirow[t]{2}{*}{ LF nu } & Rho & -0.211 & -0.069 & -0.204 & 0.064 & -0.119 & 0.138 & -0.062 & 0.116 & 0.015 \\
\hline & $p$-Value & 0.273 & 0.722 & 0.290 & 0.743 & 0.539 & 0.474 & 0.750 & 0.549 & 0.939 \\
\hline \multirow[t]{2}{*}{ HF nu } & Rho & 0.211 & 0.069 & 0.204 & -0.064 & 0.119 & -0.138 & 0.062 & -0.116 & -0.015 \\
\hline & $p$-Value & 0.273 & 0.722 & 0.290 & 0.743 & 0.539 & 0.474 & 0.750 & 0.549 & 0.939 \\
\hline \multirow[t]{2}{*}{$\mathrm{LF} / \mathrm{HF}$} & Rho & -0.195 & -0.142 & -0.206 & 0.019 & -0.129 & 0.173 & 0.123 & 0.103 & 0.020 \\
\hline & $p$-Value & 0.312 & 0.462 & 0.284 & 0.923 & 0.503 & 0.368 & 0.527 & 0.594 & 0.918 \\
\hline \multirow[t]{2}{*}{ SDI } & Rho & -0.055 & 0.013 & 0.062 & -0.118 & -0.041 & -0.069 & -0.136 & -0.056 & 0.000 \\
\hline & $p$-Value & 0.777 & 0.946 & 0.750 & 0.544 & 0.832 & 0.723 & 0.482 & 0.773 & 0.999 \\
\hline \multirow[t]{2}{*}{ SD2 } & Rho & 0.166 & 0.067 & -0.015 & -0.058 & -0.152 & -0.238 & -0.245 & -0.232 & 0.087 \\
\hline & $p$-Value & 0.389 & 0.731 & 0.939 & 0.764 & 0.430 & 0.214 & 0.200 & 0.226 & 0.653 \\
\hline \multirow[t]{2}{*}{ SDI/SD2 } & Rho & -0.087 & 0.037 & $0.28 I$ & -0.054 & 0.131 & 0.271 & 0.127 & 0.274 & -0.146 \\
\hline & $p$-Value & 0.655 & 0.850 & 0.140 & $0.78 \mathrm{I}$ & 0.500 & 0.154 & 0.512 & 0.150 & 0.451 \\
\hline
\end{tabular}

Abbreviations: BMI, body mass index; PR, pulse rate (beats/min); DBP, diastolic blood pressure; FS, fasting blood sugar; HC, hip circumference; HF, high frequency; FH nu, high frequency normalized unit; HRV, heart rate variability; LF, low frequency; LF/HF, low frequency:high frequency ratio; LF nu, low frequency normalized unit; NN50 count, number of RR intervals that differ by $>50 \mathrm{~ms}$; PNN50\%, percentage of consecutive RR intervals that differ by $>50$ ms; RMSSD, root mean square of differences of successive RR intervals; SBP, systolic blood pressure; SDI, Poincare plot SDI; SDI/SD2, SDI:SD2 ratio; SD2, Poincare plot SD2; SDNN, standard deviation of all RR intervals; WC, waist circumference; WHR, waist-hip ratio.

function as indicated by increased HF power. Also, several studies of obesity in children and adolescents have also found that vagal function is reduced in obese individuals compared to nonobese individuals. ${ }^{26-28}$ In all of these studies, several indices of vagal function, such as HF power, were reduced in the obese individuals.

In contrast to our study, a reduction in sympathetic activity in obese people has also been described in some reports..$^{29,30}$ This variation among the studies is partially explained on the basis of the duration of obesity. It has been said that the duration of obesity has a major role to play in determining the level of cardiac sympathetic activity. ${ }^{31}$ The present study showed an increase in sympathetic activity, but long-standing obesity is likely to lead to overall reduction of autonomic activity and, hence, also a reduction in sympathetic activity. ${ }^{31}$

Moreover, correlation analysis showed that the HRV parasympathetic indicators, that is, SDNN, RMSSD, NN50, $\mathrm{HF} \mathrm{ms}^{2}$, HF nu, and SD1, were inversely related with age in obese persons. Similarly, SD2 and LF $\mathrm{ms}^{2}$ were also inversely related, but LF nu and LF/HF ratio (cardiac sympathetic markers) were directly related with age in obese persons. None of the HRV measures showed significant correlation with age in the normal weight group. Thus, it is obvious from our study that in obese people, with aging, there is an increase in sympathetic activity and a simultaneous decrease in parasympathetic activity. However, at the same time, there is relative decrease in both sympathetic and parasympathetic activities with age in obese people. The findings obtained by Kim et $\mathrm{al}^{20}$ and Moodithaya and Avadhany ${ }^{32}$ are in agreement with our finding that parasympathetic activity decreases with age in obese people.

Thus, the above discussion emphasizes the finding that obesity provokes a reduction in vagal tone coupled with an increase in cardiac sympathetic activity, despite some controversies existing regarding sympathetic activity. For analyzing our findings and related reports, it was important to find out the relative association of obesity indices with the HRV indices, a novel research of its kind. In our present study, the groups (obese and normal weight) were defined solely on the basis of BMI, though we had measured and calculated other obesity indices as well. Surprisingly, on correlation analysis, BMI showed a very weak association with HRV measures in obese subjects, showing negative relation with SDNN, a parasympathetic HRV marker, which was not 
statistically significant. However, in the obese group, WHR was found to be correlated inversely and the correlation was statistically significant as well with HF powers: $\mathrm{HF} \mathrm{ms}^{2}$ and HF nu (cardiac parasympathetic indicators). It was related directly to LF power (LF nu) and LF/HF ratio (sympathetic markers) in obese subjects. In contrast, we did not find any significant correlation between WHR and HRV measures in the normal weight group. Moreover, the relationship between WC and HRV indices in obese subjects, though statistically not significant, was not of much difference to that of WHR and HRV markers. Thus, our findings showed that WC and especially WHR had a direct relation with the sympathetic nervous system and an inverse relation with the parasympathetic nervous system in obese persons, which is also supported by a previous report. ${ }^{22}$ This could possibly be true because increased WHR leads to obesity and obesity modulates the cardiac autonomic nervous system. A number of studies published previously provide evidence that there is a significantly lower parasympathetic activity ${ }^{13,4,19,22}$ and an increased sympathovagal balance ${ }^{33,34}$ in obese adolescents who have high WHR, but there are no correlation studies on this. In contrast to this study, it was reported that sympathovagal activity decreased with obesity in postmenopausal women. ${ }^{35} \mathrm{BMI}$ has traditionally been the chosen indicator to measure body size and composition and to diagnose underweight and overweight conditions. It is obvious from our findings that WHR has a stronger association with HRV markers than BMI, leading to modulation of cardiac autonomic activity. The presence of a stronger association between WC and WHR with HRV indices in comparison to BMI could be attributed to the different pattern of obesity existing in this population. BMI, however, suffers from two major drawbacks in that it does not differentiate between body fat and fat-free compartments. Therefore, a high BMI may indicate either increased fat or fat-free mass, though body composition being affected by ethnicity, growth patterns, socioeconomic, cultural, and behavioral patterns with the same BMI in people of different ethnicities and background may reflect different fat content and distribution. ${ }^{36}$ These have been developed by western researchers based on studies in Caucasian populations and in no way are designed to be applicable to all populations. The alternative measures that reflect abdominal adiposity, such as WC and WHR, have also been suggested as being superior to BMI in predicting CVD risk,$^{37}$ which is in line with our study. This is based largely on the rationale that increased visceral adipose tissue is associated with a range of metabolic abnormalities, including decreased glucose tolerance, reduced insulin sensitivity, and adverse lipid profiles, which are the risk factors for type 2 diabetes and CVD.$^{38}$ Central or visceral obesity (abdominal obesity), which is more common in Asian populations, can simply be assessed by measurement of WC and WHR. ${ }^{39}$ Abdominal obesity is considered to be more dangerous than general obesity because the visceral fat has been shown to secrete certain cytokines and chemicals that are involved in atherogenesis and alterations in the autonomic balance. ${ }^{40}$

\section{Conclusion}

Obesity increased the sympathetic activity with a reduction in parasympathetic (vagal) tone, indicating poor autonomic cardiac rhythm control in obese individuals. Increased WHR, by far an indicator of visceral adiposity, was strongly associated with reduced cardiac parasympathetic and increased sympathetic activity in obese individuals defined by BMI. However, the BMI itself has a weak degree of relationship with HRV cardiac autonomic markers. Thus, even with a slight increase in WHR in an individual, there could be a greater risk of cardiovascular morbidity and mortality brought about by cardiac autonomic alterations. Therefore, it would be wise to consider WHR as a stronger obesity marker than BMI in this population.

\section{Disclosure}

The authors report no conflicts of interest in this work.

\section{References}

1. Marie Ng, Fleming T, Robinson M, et al. Global, regional, and national prevalence of overweight and obesity in children and adults during 1980-2013: a systematic analysis for the global burden of disease study 2013. Lancet. 2014;384(9945):766-781.

2. Kaufman CL, Kaiser DR, Steinberger J. Kelly AS, Dengel DR. Relationships of cardiac autonomic function with metabolic abnormalities in childhood obesity. Obesity (Silver Spring). 2007;15(5):1164-1171.

3. Tonhajzerova I, Javorka M, Trunkvalterova Z, et al. Cardio-respiratory interaction and autonomic dysfunction in obesity. J Physiol Pharmacol. 2008;59(Suppl 6):709-718.

4. Heart rate variability: standards of measurement, physiological interpretation and clinical use. task force of the European society of cardiology and the NorthAmerican Society of Pacing andElectrophysiology. Circulation. 1996;93(5):1043-1065.

5. Vanderlei LCM, Pastre CM, Hoshi RA, Carvalho TD, Godoy MF. Basic notions of heart rate variability and its clinical applicability. Rev Bras Cir Cardiovasc. 2009;24(2):205-217.

6. Rolim LC, de Souza JST, Dib SA. Tests for early diagnosis of cardiovascular autonomic neuropathy: critical analysis and relevance. Front Endocrinol (Lausanne). 2013;4:173.

7. Laederach-Hofmann K, Mussgay L, Ruddel H. Autonomic cardiovascular regulation in obesity. J Endocrinol. 2000;164(1):59-66.

8. Hurt RT, Kulisek C, Buchanan LA, McClave SA. The obesity epidemic: challenges, health initiatives, and implications for gastroenterologists. Gastroenterol Hepatol (N Y). 2010;6(12):780-792.

9. Romero-Corral A, Somers VK, Sierra-Johnson J, et al. Accuracy of body mass index to diagnose obesity in the US adult population. Int $J$ Obes (Lond). 2008;32(6):959-966.

10. World Health Organization: obesity and overweight. Fact sheet No. 311, [updated June 2016]. Available from: http://www.who.int/mediacentre/ factsheets/fs311/en/. Accessed December 13, 2016. 
11. Chobanian AV, Bakris GL, Black HR, et al. Seventh report of the Joint National Committee on Prevention, Detection, Evaluation, and Treatment of High Blood Pressure. Hypertension. 2003;42(6):1206-1252.

12. World Health Organization. Waist Circumference and Waist-Hip Ratio: Report of a WHO Expert Consultation. Geneva, Switzerland: World Health Organization; 2008:8-11.

13. Rabbia F, Silke B, Conterno A, et al. Assessment of cardiac autonomic modulation during adolescent obesity. Obes Res. 2003;11(4): 541-548.

14. Freitas IM, Miranda JA, Mira PA, Lanna CM, Lima JR, Laterza MC. Cardiac autonomic dysfunction in obese normotensive children and adolescents. Rev Paul Pediatr. 2014;32(2):244-249.

15. Nagai N, Matsumoto T, Kita H, Moritani T. Autonomic nervous system activity and the state and development of obesity in Japanese school children. Obes Res. 2003;11(1):25-32.

16. Khrisanapant W, Sengmeuang P, Pasurivong O, Kukongviriyapan U. Does cardiac autonomic modulation exist in obese adolescents? Srinagarind Med J. 2008;23(3):234-239.

17. Theodore A. Kotchen. Obesity-related hypertension: epidemiology, pathophysiology, and clinical management. Am JHypertens. 2010;23(11): $1170-1178$.

18. Huxley R, Mendis S, Zheleznyakov E, Reddy S, Chan J. Body mass index, waist circumference and waist: hip ratio as predictors of cardiovascular risk - a review of the literature. Eur J Clin Nutr. 2010;64(1): $16-22$.

19. Billman GE, Huikuri HV, Sacha J, Trimmel K. An introduction to heart rate variability: methodological considerations and clinical applications. Front Physiol. 2015;6:55.

20. Kim JA, Park YG, Cho KH, Hong MH, Han HC, Choi YS, Yoon D. Heart rate variability and obesity indices: emphasis on the response to noise and standing. J Am Board Fam Pract 2005;18(2):97-103.

21. Thorp AA, Schlaich MP. Relevance of sympathetic nervous system activation in obesity and metabolic syndrome. J Diabetes Res. 2015;2015:Article ID 341583.

22. Vanderlei LCM, Pastre CM, Freitas Jr IF, Godoy MF. Analysis of cardiac autonomic modulation in obese and eutrophic children. Clinics (Sao Paulo). 2010;65(8):789-792.

23. Esler M, Straznicky N, Eikelis N, Masuo K, Lambert G, Lambert E. Mechanisms of sympathetic activation in obesity-related hypertension. Hypertension. 2006;48(5):787-796.

24. Petretta M, Bonaduce D, de Filippo E, et al. Assessment of cardiac autonomic control by heart period variability in patients with early-onset familial obesity. Eur J Clin Invest. 1995;25(11):826-832.

25. Karason K, Molgaard H, Wikstrand J, Sjostrom L. Heart rate variability in obesity and the effect of weight loss. Am J Cardiol. 1999;83(8): $1242-1247$
26. Riva P, Martini G, Rabbia F, Milan A, Paglieri C, Chiandussi L, Veglio F. Obesity and autonomic function in adolescence. Clin Exp Hypertens. 2001;23(1-2):57-67.

27. Nagai N, Matsumoto T, Kita H, Moritani T. Autonomic nervous system activity and the state and development of obesity in Japanese school children. Obes Res. 2003;11(1):25-32.

28. Thayer JF, Yamamoto SS, Brosschot JF. The relationship of autonomic imbalance, heart rate variability and cardiovascular disease risk factors. Int J Cardiol. 2009; 141(2):122-131.

29. Yakinci C, Mungen B, Karabiber H, Tayfun M, Evereklioglu C. Autonomic nervous system functions in obese children. Brain Dev. 2000;22(3):151-153.

30. Nagai N, Moritani T. Effect of physical activity on autonomic nervous system function in lean and obese children. Int $J$ Obesity. 2004; 28(1):27-33.

31. Gutin B, Barbeau P, Litakar MS, Ferguson M, Owens S. Heart rate variability in obese children: relations to total body and visceral adiposity, and changes with physical training and detraining. Obes Res. 2000;8(1):12-19.

32. Moodithaya S, Avadhany ST. Gender differences in age-related changes in cardiac autonomic nervous function. J Aging Res. 2012;2012:679345.

33. Martini G, Riva P, Rabbia F, et al. Heart rate variability in childhood obesity. Clin Auton Res. 2002;11(2):87-91.

34. Guizar JM, Ahuatzin R, Amador N, Sa'nchez G, Romer G. Heart autonomic function in overweight adolescents. Indian Pediatr. 2005; 42(5):464-469.

35. Chaudhuri A, Borade NG, Tirumalai J, Saldanha D, Ghosh B, Srivastava $\mathrm{K}$. A study of autonomic functions and obesity in postmenopausal women. Ind Psychiatry J. 2012;21(1):39-43.

36. Rothman KJ. BMI-related errors in the measurement of obesity. Int $J$ Obes. 2008;32(Suppl 3):S56-S59.

37. Ahmad N, Adam SIM, Nawi AM, Hassan MR, Ghazi HF. Abdominal obesity indicators: waist circumference or waist-to-hip ratio in Malaysian adults population. Int J Prev Med. 2016;7:82.

38. Lam BCC, Koh GCH, Chen C, Wong MTK, Fallows SJ. Comparison of body mass index (BMI), body adiposity index (BAI), waist circumference (WC), waist-to-hip ratio (WHR) and waist-to-height ratio (WHtR) as predictors of cardiovascular disease risk factors in an adult population in Singapore. PLoS One. 2015;10(4):e0122985.

39. Hurt RT, Kulisek C, Buchanan LA, McClave SA. The obesity epidemic: challenges, health initiatives, and implications for gastroenterologists. Gastroenterol Hepatol (N Y). 2010;6(12):780-792.

40. Prasad DS, Kabir Z, Dash AK, Das BC. Abdominal obesity, an independent cardiovascular risk factor in Indian subcontinent: a clinico epidemiological evidence summary. J Cardiovasc Dis Res. 2011;2(4): 199-205.
Diabetes, Metabolic Syndrome and Obesity: Targets and Therapy is an international, peer-reviewed open-access journal committed to the rapid publication of the latest laboratory and clinical findings in the fields of diabetes, metabolic syndrome and obesity research. Original research, review, case reports, hypothesis formation, expert opinion and commentaries are all considered for publication. The manuscript management system is completely online and includes a very quick and fair peer-review system, which is all easy to use. Visit http://www.dovepress.com/testimonials.php to read real quotes from published authors. 